\title{
Characterization of an Ebosin derivative produced by heterologous gene replacement in Streptomyces sp. 139
}

\author{
Yang Zhang ${ }^{1 \dagger}$, Junjie Shan ${ }^{2 \dagger}$, Yonggang Bao $^{1 \dagger}$, Liping Bai ${ }^{1}$, Rong Jiang ${ }^{1}$, Lianhong Guo ${ }^{1}$, Chen Yao ${ }^{1}$,
} Ren Zhang ${ }^{3}$ and Yuan $\mathrm{Li}^{i^{*}}$

\begin{abstract}
Background: Ebosin is a novel exopolysaccharide (EPS) produced by Streptomyces sp. 139 and evidenced to possess an anti-rheumatic arthritis activity in vivo. The Ebosin biosynthesis gene cluster (ste) consists of 27 ORFs and ste7 has previously been demonstrated to code for a fucosyltransferase, which plays an essential role in the formation of repeating sugar units during Ebosin production. Aiming to generate derivatives of Ebosin for better activity, we replaced ste7 with a gene encoding for a glucosyltransferase ( $g$ tf from Streptococcus thermophilus.

Results: This alteration resulted in a novel Ebosin derivative (EPS-7 g) with its monosaccharide composition dramatically changed, especially in the proportion of glucose which increased from 1.1\% (Ebosin) to 84.01\% (EPS-7 g). In an ELISA analysis, EPS-7 g exhibited a higher binding activity for IL-1R, as a competitor of interleukin-1, than that of Ebosin. It also exhibited a higher inhibitory effect on the activity of IL-1 $\beta$-converting enzyme and production of IL-1 $\beta$ in fibroblast-like synoviocytes (FLS). In addition, experiments with acute inflamed mice induced by croton oil showed a significantly higher anti-inflammatory activity of EPS-7 g compared with Ebosin.
\end{abstract}

Conclusions: The new Ebosin derivative EPS-7 $\mathrm{g}$ is more bioactive than Ebosin evaluated by a series of experiments. This is the first report demonstrating a modification of EPS structure via heterologous gene replacement in Streptomyces.

Keywords: Ebosin derivative, Heterologous gene replacement, Gene gtf, Gene ste7, Streptomyces, Streptococcus thermophilus

\section{Background}

One type of microbial polysaccharides are secreted out of the cells [1] and therefore called exopolysaccharides (EPSs), which are long-chain polysaccharides consisting of branched, repeating units of sugars or sugar derivatives [2]. In bacteria, EPS biosynthesis starts with the intracellular formation of EPS precursors and the sugar nucleotides, followed by the formation of a repeating unit on a lipid carrier which is located in the cytoplasmic membrane. The later steps involve transport of the repeating units across the membrane to the outer layer

\footnotetext{
* Correspondence: yuanwli@263.net

${ }^{\dagger}$ Equal contributors

'Key laboratory of Biotechnology of Antibiotics, Ministry of Health, Institute of Medicinal Biotechnology, Chinese Academy of Medical Sciences \& Peking Union Medical College, Tian Tan, 100050 Beijing, China

Full list of author information is available at the end of the article
}

and polymerization of tens and even hundreds of such units to form the final EPSs [3]. During the process, glycosyltransferases play important roles to sequentially transfer sugars from intracellular nucleotide sugars to a lipid carrier acceptor [4].

In order to improve applications of EPSs, genetic engineering can be used in the production of desired polysaccharides targeting predefined macroscopic properties.

Based on the available genetic information, genetic modification of eps genes should lead to EPSs with a different repeating unit or with a different chain length [5]. Manipulation of genes which function in export, polymerization, and determination of chain length during EPS biosynthesis were also proved effective for altering EPS structure [6]. Heterologous production of an EPS has been successful by transfer of the complete eps gene cluster alone of a LAB strain into a non EPS-producing 
heterologous host, provided that the heterologous host possessed all necessary genetic information for precursor synthesis [7]. Ingeborg et al [8] described increased exopolysaccharide production in Lactoccus lactis due to manipulated overexpression of the NIZO B40 eps gene cluster, the first report demonstrating that homologous overexpression of a complete eps gene cluster in Lactoccus lactis leads to increased EPS production.

Streptomyces are a group of gram-positive bacteria that have been intensively studied for their secondary metabolites, particularly antibiotics. However, little is known of the production of EPSs in Streptomyces. Recently a novel EPS namely Ebosin was isolated from the supernatants of fermentation cultures of Streptomyces sp. 139 [9], which has remarkable anti-rheumatic arthritis activity in vivo [10]. Its biosynthesis gene cluster (ste) consisting of 27 ORFs was also identified [11]. Efforts have been made in elucidating the function of individual ste genes [12-15]. Insights into the biosynthesis pathways of polysaccharides are crucial for the exploitation of microorganisms to produce polysaccharides of industrial or medicinal importance. With a number of the ste genes identified, it is now possible to carry out specific manipulations for creating Ebosin derivatives which can then be screened for better bioactivities. This paper reports our endeavor to generate such derivatives by tackling the ste 7 gene encoding a fucosyltransferase, which catalyzes the transfer of fucose specifically from GDP-fucose to a fucose acceptor during Ebosin biosynthesis [16]. In this study, we replaced ste7 with the glucosyltransferase gene ( $g t f$ ) from Streptococcus thermophilus, which is a gram-positive bacterium and most valuably used in food industry. Stingele et al [17] identified the eps genetic locus of S. thermophilus Sfi6, revealing a $15.25-\mathrm{kb}$ region containing 16 open reading frames (ORFs), within a 14.52-kb region encodes 13 genes (epsA to epsM) capable of directing EPS synthesis. The resultant EPS-7 g produced by the strain Streptomyces sp. 139 (gtf) has a very high content of glucose and exhibited remarkably higher bioactivities than that of Ebosin in both in vitro and in vivo assays.

\section{Results}

\section{Construction of the heterologous gene replacement} strain Streptomyces sp. 139 (gtf)

The replacement of $s t e 7$ gene by the gtf gene of $S$. thermophilus (Figure 1A) was evidenced by Southern hybridization using a 1.03-kb DNA (F1) as probe upstream of ste7 (Figure 1B). The hybridization signals appeared with the expected sizes of $4.0 \mathrm{~kb}$ for Streptomyces sp. 139 (gtf) and $3.1 \mathrm{~kb}$ for Streptomyces sp. 139 (ste7). This result indicated that the colonies with thio ${ }^{\mathrm{r}} \mathrm{Am}^{\mathrm{s}}$ phenotypes had integrated the $g t f-$ thio $^{r}$ cassette into the kanamycin resistance gene, therefore, confirmed the establishment of heterologous gene replacement strain Streptomyces sp. 139 (gtf).

\section{Sugar composition of EPSs}

GC analysis of Ebosin, EPS-7 m produced by the knock mutant Streptomyces sp. 139 (ste7) and EPS-7 g by Streptomyces sp. 139 (gtf) (Figure 2A, B, C) showed that these EPSs consisted of the same monosaccharides: glucose, mannose, arabinose, galactose, fucose, xylose and rhamnose, of which the most striking was an increase of glucose proportion from 1.1\% (Ebosin) to $84.01 \%$ (EPS-7 g). While, some proportional changes of other sugars also occurred in comparison with Ebosin (Figure 2D). Using a method based on uronic acid carbazole reaction, galacturonic acid was also found in EPS-7 m and EPS-7 g.

\section{The competitive binding activity of Ebosin derivates with} IL-1 for IL-1R

Using an ELISA assay, the competitive binding activities of Ebosin, EPS-7 $\mathrm{m}$ and EPS-7 $\mathrm{g}$ with IL-1 for IL-1R were determined.

For EPS-7 $\mathrm{m}$ they were $6.1 \%$ and nil $(P<0.05)$ at dosages of $0.64 \mathrm{ng} / \mu \mathrm{L}$ and $0.128 \mathrm{ng} / \mu \mathrm{L}$ respectively, which were remarkably lower than those of Ebosin (24.2\%, $13.7 \%)$ at the same dosages. Higher binding activities of EPS-7 $\mathrm{g}$ were detected to be $27.1 \%$ and $24.3 \%(P<0.05)$ respectively compared with that of Ebosin at same concentrations (Figure 3).

\section{Effect of Ebosin derivates on the enzymatic activity for} IL-1 $\beta$-converting enzyme (ICE)

At concentrations of $0.64 \mathrm{ng} / \mu \mathrm{L}$ and $0.128 \mathrm{ng} / \mu \mathrm{L}$, Ebosin suppressed the enzyme with inhibition ratios $35.5 \%$ and $25.0 \%$ respectively, compared with $15.5 \%(P<0.05), 14.3 \%$ for EPS-7 $\mathrm{m}$ and $47.6 \%, 35.7 \%(P<0.05)$ for EPS-7 $\mathrm{g}$ respectively (Figure $4 \mathrm{~A})$. The results demonstrated a higher activity of EPS-7 $g$ to inhibit the IL-1 $\beta$-converting enzyme.

\section{Inhibiting effect of Ebosin derivates on production of IL- $1 \beta$ in FLS cells}

To assess the effects of Ebosin, EPS-7 $\mathrm{m}$ and EPS-7 $\mathrm{g}$ on production of IL-1 $\beta$ in FLS (fibroblast-like synoviocytes), cell cultures were carried out and stimulated with LPS at $37^{\circ} \mathrm{C}$ for $72 \mathrm{~h}$ before analyzed by ELISA. The results indicated (Figure 4B) that Ebosin, EPS-7 $\mathrm{m}$ and EPS-7 $\mathrm{g}$ at dosage of $3.2 \mathrm{ng} / \mu \mathrm{L}$ reduced the IL-1 $\beta$ production 52.03\% $(P<0.01), 26.17 \%, 58.70 \%(P<0.01)$ respectively and $45.57 \%(P<0.5), 15.39 \%, 52.34 \%(P<0.01)$ at dosage of $0.64 \mathrm{ng} / \mu \mathrm{L}$ separately. With dosage of $0.128 \mathrm{ng} / \mu \mathrm{L}$, the IL- $1 \beta$ production were suppressed $31.61 \%, 10.16 \%$ and $47.82 \%(P<0.01)$ individually.

Effect of Ebosin and EPS-7 $\mathrm{g}$ on the inflammatory activity in the acute inflamed mice induced by croton oil

To evaluate the effect of Ebosin and EPS-7 $g$ on the acute inflamed mice induced by croton oil, the mice were randomly divided into three groups described as above. Ebosin 


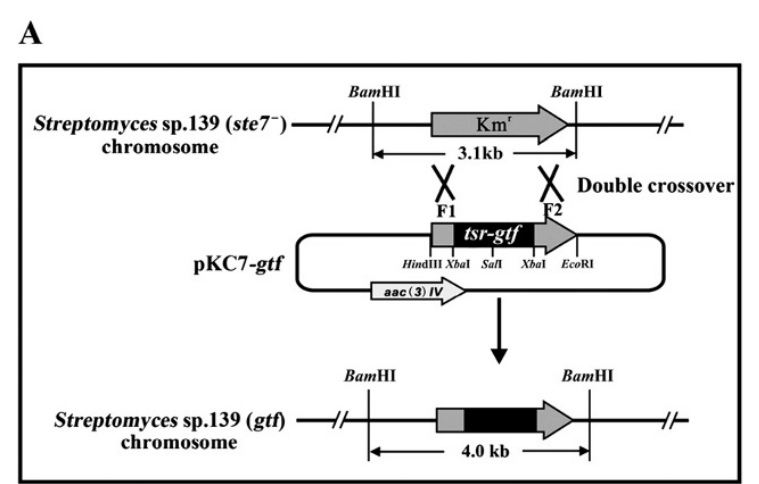

B

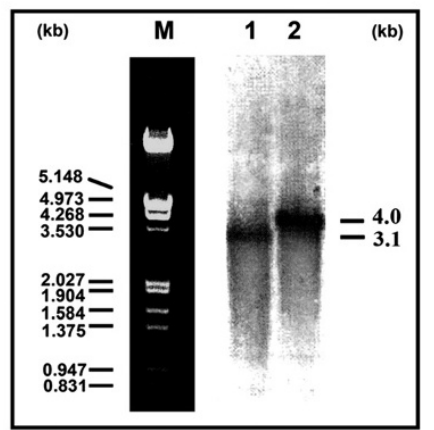

Figure 1 The diagram of gene replacement and Southern blot analysis. (A) Diagram of gene replacement of ste7 with gtf originated from Streptococcus thermophilus through a double crossover via homologous recombination. Gray box indicates the location of $\mathrm{Km}^{r} \mathrm{gene}$ in Streptomyces sp. 139 (steT). Restriction maps of Streptomyces sp. 139 (steT) and the gene replacement strain Streptomyces sp. 139 (gtf) show the predicted fragment sizes upon BamHI digestion. (B) Southern blot autoradiograph of Streptomyces sp. 139 (ste7) and Streptomyces sp. 139 (gtf). 1. Chromosome DNA of Streptomyces sp. 139 (steT) digested with BamHl; 2. chromosome DNA of Streptomyces sp. 139 (gtf) digested with BamHI.

and EPS-7 g $(100 \mathrm{mg} / \mathrm{kg})$ were orally administered to each mouse of the respective experimental groups for $1 \mathrm{~h}$ before induction by croton oil. Compared with the acute inflamed mice groups untreated (control), the inhibition ratios of EPS-7 $\mathrm{g}$ and Ebosin for mice ear edema were $48.61 \%(P<0.05)$ and $21.60 \%$ respectively (Figure 5$)$, indicating that EPS-7 g surpasses Ebosin by over 100\% on suppressing the inflammatory activity in the acute inflamed mice.

\section{Discussion}

Exopolysaccharides include a range of diverse polymers that play vital roles in variety of biological processes. In addition, EPSs have also significant industrial applications,
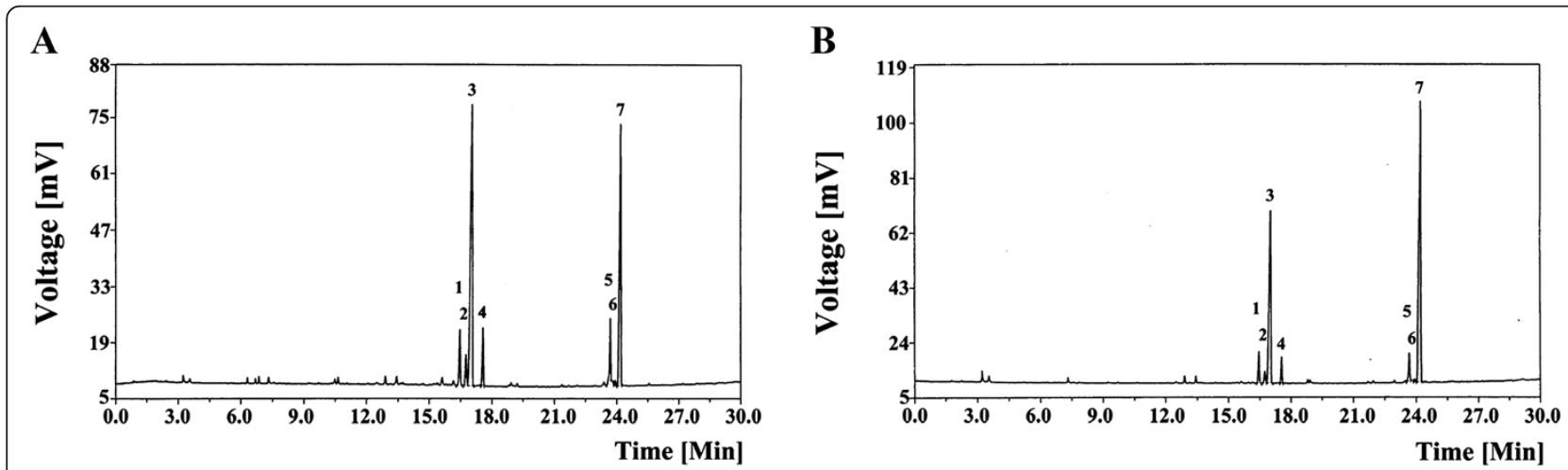

C

D
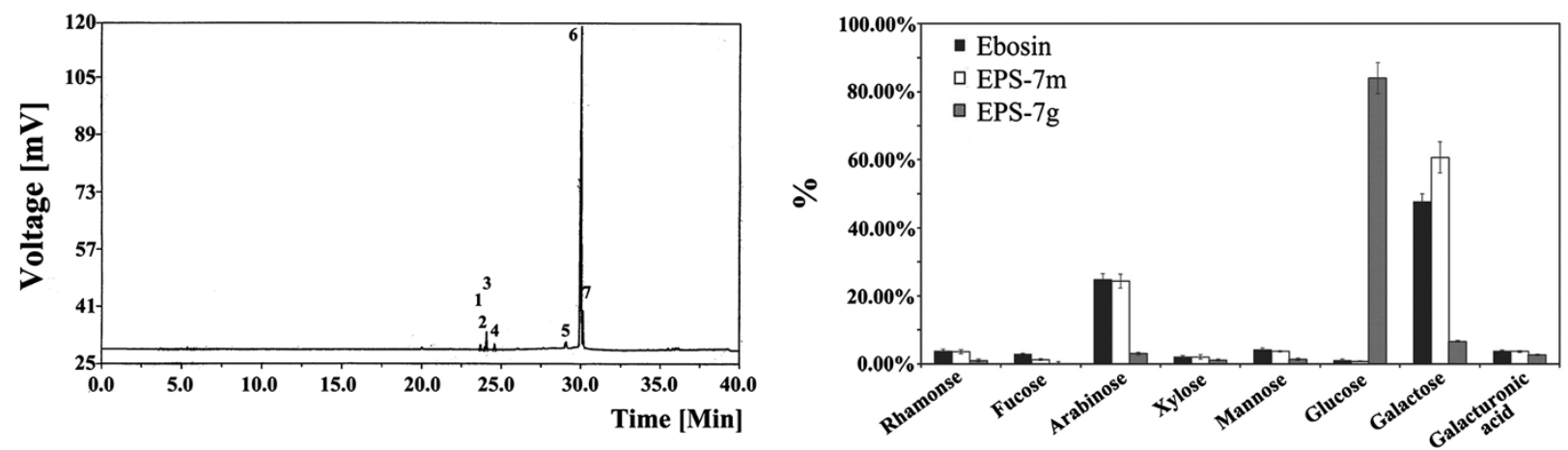

Figure 2 GC chromatograms of sugar analysis of Ebosin (A), EPS-7 m (B) and EPS-7 g (C). 1. rhamnose, 2. fucose, 3. arabinose, 4. xylose, 5. mannose, 6. glucose, 7. galactose. (D). Monosaccharide compositions of Ebosin, EPS-7 m and EPS-7 g. Galacturonic acid was analyzed using a method based on uronic acid carbazole reaction (Bitter et al., 1962). 


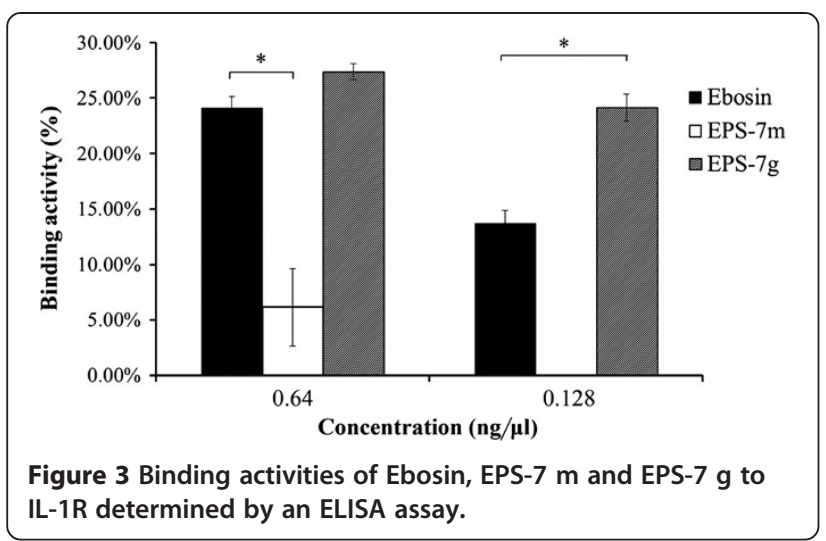

including their use as biothickeners in foods [4]. Notably, EPSs produced by lactic acid bacteria contribute significantly to the structure and viscosity of fermented milk products [18]. The health value of these macromolecules has also emerged in recent years; many reports indicate that they can confer health benefits on consumers arising from their immunogenic and cholesterol-lowering properties [19,20]. EPSs produced by Trichoderma erinaceum DG-312 was shown to have a strong antiinflammatory activity in inflamed mice [21]. Enterobacter cloacae was also found to produce EPSs with anti-diabetic activity [22].

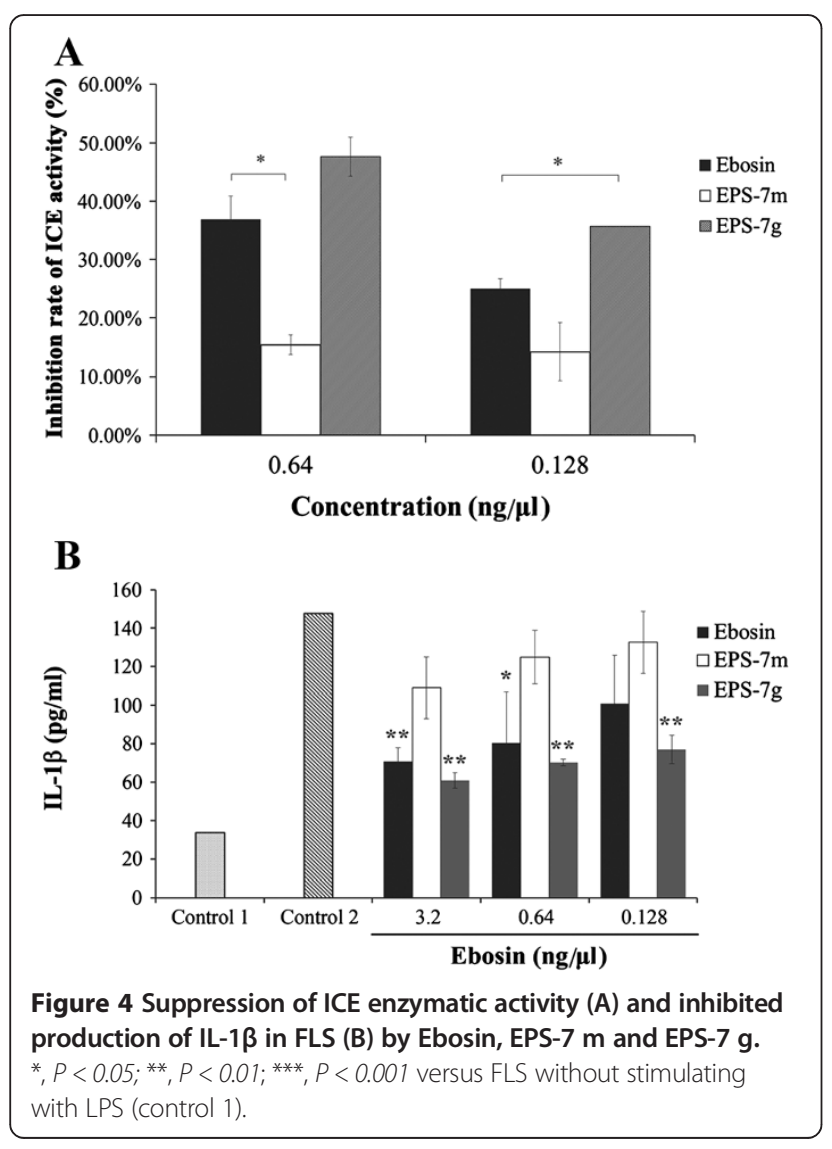

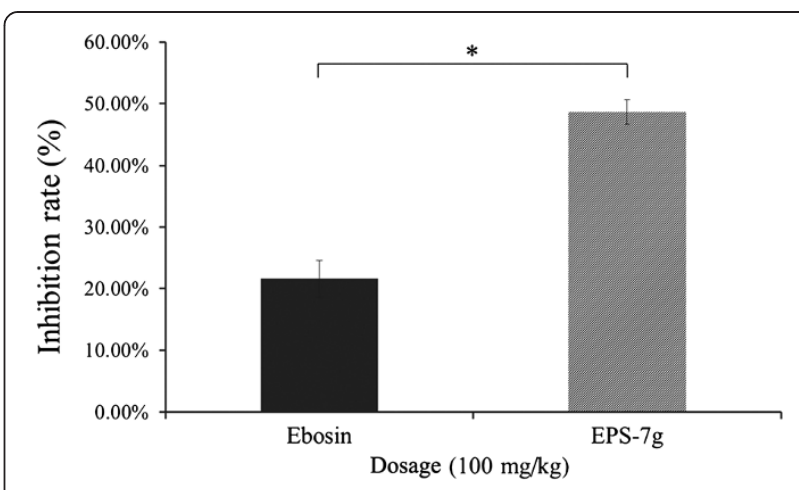

Figure 5 Effect of EPSs on inflammatory activity in the acute inflamed mice induced by croton oil.

Metabolic engineering has enabled generation of "designer" polysaccharides in lactic acid bacteria (LAB), which mostly involved manipulations of glycosyltransferases [2]. Introducing new or existing glycosyltransferases into LAB [4] or gene shuffling with glycosyltransferases are effective means for controlling EPS structure [5]. Masja et al [23] reported that heterologous production of the pneumococcal serotype 14 polysaccharide in Lactococcus lactis resulted in the recombinant product secreted into culture medium, which simplified downstream processing. This was achieved by coexpressing the pneumococcal gene cluster $c p s F G H I J K L_{14}$ with the lactococcal regulatory and priming glucosyltransferase-encoding genes (eps $\left.A B C D_{\mathrm{B} 40}\right)$ specific for B40 polysaccharide. Knoshaug et al [24] reported evidence for a novel gene organization expressing EPS in Lactococcus lactis subsp. cremoris Ropy352 and showed the specificities of polymerization and export enzymes blocking the function of just one glycosyltransferase abolished the production of ropy EPS. Heterologous expression of glycosyltransferases has been found to result in different sugars added at strategic positions in generating EPSs with new properties [5].

Ebosin is a novel EPS with anti-rheumatic arthritis activity. It has been shown that Ebosin is an inhibitor of IL-1 $\beta$-converting enzyme (ICE), a key enzyme in synthesis of IL-1 $\beta$ [25]. More recently, evidence has also been obtained demonstrating that the anti-inflammatory effect of Ebosin on rat collagen-induced arthritis is through suppressing production of interleukin- $1 \beta$, interleukin- 6 and tumor necrosis factor $\alpha$ at both transcriptional and posttranslational levels [10]. This EPS is therefore of medicinal value so improvement for better property is warranted. In this study, a glucosyltransferase gtf gene from S. thermophiles was expressed in replacement of the ste7 gene which encodes a fucosyltransferase in the Ebosin-producing strain Streptomyces sp. 139. This brought about dramatic changes in the property of EPS produced. Because of the complexity of the biosynthesis pathway of Ebosin and involvement of a large number of genes, once ste 7 was replaced with the 
heterologous gene $g t f$, dramatic effect was seen not only on the increased incorporation of glucose, but also on the overall sugar profile. The reason for these changes, which are apparently not a simple proportional change of composition with increased glucose, may lie in the primary structure. The resultant EPS-7 g turns out to be more bioactive compared with Ebosin evaluated by its ICE inhibitory activity, suppression of interleukin-1 $\beta$ production in fibroblast-like synoviocytes (FLS), competitive binding to IL-1R against IL-1 and suppression of the acute inflammatory activity in the acute inflamed mice induced by croton oil. According to these results, it looks like that increasing proportion of glucose effects on bioactivity of EPS-7 g. A good understanding of the correlation between structure and bioactivity of Ebosin is important because it will provide a foundation for a strategy aimed at producing functionally more valuable polysaccharides. Continuing efforts in the elucidation of Ebosin biosynthesis pathway will enable more rational designs for genetic manipulation to generate highly effective derivatives.

\section{Conclusions}

EPS-7 g, a novel Ebosin derivate, was produced by heterologous gene replacement in Streptomyces sp. 139, which was more bioactive compared with Ebosin evaluated by a series of experiments. To our knowledge, this is the first report on manipulation of EPS structure by introducing heterologous glucosyltransferases into Streptomyces.

\section{Methods}

\section{Bacterial strains and culture conditions}

Streptomyces sp. 139 was isolated from a soil sample in China and kept in the China General Microbiology Culture Collection Center (No. 0405) (Table 1). Streptomyces sp.
139 (ste7) was generated previously [16] and kept in our laboratory. These strains were cultured at $28^{\circ} \mathrm{C}$ with shaking $(250 \mathrm{rpm})$ in either TSB medium supplemented with $5 \mathrm{mM} \mathrm{MgCI}_{2}$ and $0.5 \%$ glycine or fermentation medium ( $1 \%$ glucose, $2 \%$ starch, $2 \%$ soybean extract, $0.2 \%$ tryptone, $0.2 \%$ beef extract, $0.4 \%$ yeast extract, $0.05 \% \mathrm{~K}_{2} \mathrm{HPO} 4,0.3 \%$ $\mathrm{CaCO}_{3}, \mathrm{pH}$ 7.3). Streptococcus thermophilus was obtained from China Industrial Microbiology Culture Collection Center (CICC 20370) (Table 1)and cultured in LB medium at $40^{\circ} \mathrm{C}$.

\section{Animal}

Kunmin mice (male, 18-20 g, Certificate No. SCXK 2005-0013) and Wistar rats (male, $180 \pm 20 \mathrm{~g}$, Certificate No.: SCXK 2005-0013) were purchased from the Institute of Experimental Animals, Chinese Academy of Medical Sciences, Beijing. All rats were housed under standard laboratory conditions with the approval of the Institute of Experimental Animals and Use Committee of Chinese Academy of Medical Sciences.

\section{Cell culture of FLS (fibroblast-like synoviocytes)}

Synovial tissues obtained from the knee joints of sacrificed CIA (collagen- induced arthritis) rats [26] on day 30 after immunization were minced and digested with type II collagenase $\left(0.4 \%\right.$, Gibco) at $37^{\circ} \mathrm{C}$ in a humidified $5 \% \mathrm{CO}_{2}$ incubator for $2 \mathrm{~h}$ in Dulbecco's modified Eagle's medium (DMEM), thoroughly washed and then cultured in DMEM supplemented with fetal bovine serum (15\%, Gibco). At confluence, adherent cells were trypsinized $\left(0.25 \%\right.$, Hyclone) at $37^{\circ} \mathrm{C}$ for $0.5 \mathrm{~h}$, filtered and extensively washed again. The adherent cells were cultured in DMEM containing fetal bovine serum, 100 units $/ \mathrm{mL}$ penicillin and $100 \mu \mathrm{g} / \mathrm{mL}$ streptomycin in a humidified

Table 1 Bacterial strains and plasmids used in this study

\begin{tabular}{|c|c|c|}
\hline Strain or plasmid & Description & Reference \\
\hline \multicolumn{3}{|l|}{ Strains } \\
\hline E.coli DH5a & supE44 $\Delta$ lacU169 ( 980 lacZ $\Delta$ M15) hsdR17 recA1 endA1 gyrA96 thi-1 relAl & {$[27]$} \\
\hline E. coli DH5a (pUC-gtf) & E. coli DH5a clone with gtf & This study \\
\hline E. coli ET12567 & Methylation-deficient E. coli damdcmhsdM & {$[29]$} \\
\hline Streptomyces sp. 139 & Ebosin producing strain & Lab stock \\
\hline Streptomyces sp. 139 (ste7) & The gene ste7 disruption mutant & This study \\
\hline Streptomyces sp. 139 (gtf) & $\mathrm{Am}^{\mathrm{s}}$, thior ${ }^{\mathrm{r}}$, the heterologous gene replacement strain of Streptomyces sp.139 & This study \\
\hline Streptococcus thermophilus & Wild type & CICC 20370 \\
\hline \multicolumn{3}{|l|}{ Plasmids } \\
\hline pUC18 & Amprr, E.coli general cloning vector with multiple cloning site & {$[27]$} \\
\hline pUC-gtf & Ampri, pUC18 derived plasmid carrying gtf gene & This study \\
\hline $\mathrm{pKC7}$ & $\mathrm{Km}^{\mathrm{r}}$, Amr, pKC1139 derived plasmid carrying F1, F2 & This study \\
\hline pKC7-gtf & pKC7 carrying gtf gene & This study \\
\hline pEGM-T-tsr & pEGM-T carrying thio gene & Lab stock \\
\hline
\end{tabular}


atmosphere of $5 \% \mathrm{CO}_{2}$ incubator for $24 \mathrm{~h}$. FLS from passage 3-4 were seeded at $1 \times 10^{6} / \mathrm{mL}$ in 24-well plates (Nunc) or at $1 \times 10^{5} / \mathrm{mL}$ in 6-well plates in DMEM and cultivated at $37^{\circ} \mathrm{C}$ for $24 \mathrm{~h}$.

\section{DNA isolation and southern blot analysis}

Isolation of E. coli plasmid DNA, S. thermophilus genomic DNA and standard recombinant DNA techniques were performed as described by Sambrook and Russell [27]. Streptomyces plasmid and genomic DNA was isolated as mentioned by Kieser et al [28]. For Southern blot analysis, the DIG high prime DNA labeling and detection starter kit II obtained from Roche (USA) was used following the instructions of manufacturer.

\section{Cloning of the gene gtf of Streptococcus thermophilus}

The genomic DNA isolated from 48-hour cultured $S$. thermophilus was used as template. The gtf gene was amplified by PCR using primers P1 and P2 (Table 2) in the following conditions: an initial denaturation at $94^{\circ} \mathrm{C}$ for $10 \mathrm{~min}$; 30 cycles of $1 \mathrm{~min}$ at $94^{\circ} \mathrm{C}, 1 \mathrm{~min}$ at $47^{\circ} \mathrm{C}$ and $3 \mathrm{~min}$ at $72^{\circ} \mathrm{C}$; and finally $10 \mathrm{~min}$ at $72^{\circ} \mathrm{C}$. The amplified DNA fragment was cloned into plasmid pUC18 digested with EcoRI and SalI to construct pUC-gtf. The correct nucleotide sequence of $g t f$ gene fragment cloned in pUC-gtf was verified by sequencing using an $\mathrm{ABI}$ PRISM 377XL DNA Sequencer (Applied Biosystems). Then the recombinant plasmid pUC-gtf was transformed into the strain E. coli $\mathrm{DH} 5 \alpha$ to produce the strain E. coli DH5 $\alpha$ (pUC-gtf). This plasmid pUC-gtf was isolated according the protocol mentioned by Sambrook and Russell [27].

\section{Construction of the strain Streptomyces sp. 139 (gtf)}

With the plasmid pEGM-T-tsr (unpublished data) as template, the thiostrepton resistant gene $\left(\right.$ thio $\left.^{r}\right)$ was amplified by PCR using primers P3 and P4 (Table 2) in the following conditions: an initial denaturation at $94^{\circ} \mathrm{C}$ for $5 \mathrm{~min} ; 30$ cycles of $1 \mathrm{~min}$ at $94^{\circ} \mathrm{C}, 0.5 \mathrm{~min}$ at $58^{\circ} \mathrm{C}$ and $2 \mathrm{~min}$ at $72^{\circ} \mathrm{C}$; and finally $10 \mathrm{~min}$ at $72^{\circ} \mathrm{C}$. The amplified $1.06 \mathrm{~kb}$ gene fragment $(\text { thio })^{\mathrm{r}}$ ) was inserted into plasmid pUC-gtf digested with SalI- HindIII, which was then digested by $X b a \mathrm{I}$ to isolate the fragment of thio ${ }^{\mathrm{r}}-\mathrm{gtf}$. During construction of the ste 7 gene disrupted strain Streptomyces sp. 139 (ste 7 ) in previous study [16], the gene ste7 disruption vector $\mathrm{pKC} 7$ containing a 1.03-kb F1

Table 2 Oligonucleotide primers used in this study

\begin{tabular}{ll}
\hline Primer & Sequence $\mathbf{5}^{\prime} \mathbf{-} \mathbf{3}^{\prime}$ \\
\hline P1 & 5'-GCGAATTC TCTAGAATGGCGTGGCTAATTAAATG-3' \\
P2 & 5'-GCGTCGAGTTAATCGCTTCAATA-3' \\
P3 & 5'-GCGTCGACAGGCGAATACTTCATATG-3' \\
P4 & 5'-GCAAGCTT TCTAGATGATCATCACTGACGAAT-3' \\
\hline
\end{tabular}

fragment upstream of the gene ste7 and a F2 0.899-kb fragment downstream of the gene ste 7 was created. The fragment of thio ${ }^{\mathrm{r}}-\mathrm{gtf}$ was introduced into the ste 7 disruption vector $\mathrm{pKC} 7$ at $\mathrm{XbaI}$ site to construct the gene replacement plasmid pKC7-gtf. Propagated in the methylation-deficient E. coli 12567 [29], pKC7-gtf was isolated and introduced into Streptomyces sp. 139 (ste 7 ) by polyethylene glycol (PEG)-mediated protoplast transformation [28]. Incubated at $28^{\circ} \mathrm{C}$ for 16 to $20 \mathrm{~h}$, the plates were overlaid with soft R2YE $(0.7 \%$ agar) containing apramycin $(40 \mu \mathrm{g} / \mathrm{mL})$. Plasmid pKC7gtf bears a temperature-sensitive Streptomyces replication origin [20] that is unable to replicate at temperatures above $34^{\circ} \mathrm{C}$. Therefore, the transformants were first incubated at $28^{\circ} \mathrm{C}$ for 2 days until pinpoint size colonies became visible and then shifted to $37^{\circ} \mathrm{C}$ for further incubation. Strains resulted from a double crossover via homologous recombination grew out of the original pinpoint-size colonies in several days. The heterologous gene replacement strain Streptomyces sp. 139 (gtf) was selected by both apramycin sensitivity $\left(\mathrm{Am}^{\mathrm{s}}, 40 \mu \mathrm{g} / \mathrm{mL}\right)$ and thiostrepton resistance (thio ${ }^{\mathrm{r}}, 50 \mu \mathrm{g} / \mathrm{mL}$ ).

Five thio ${ }^{\mathrm{r}} \mathrm{Am}^{\mathrm{s}}$ colonies were selected randomly and the isolated genomic DNA originated from Streptomyces sp. 139 (gtf) and Streptomyces sp. 139 (ste7) [16] were digested with BamHI individually before DNA blot analysis.

\section{Isolation of EPSs}

The strains of Streptomyces sp. 139, Streptomyces sp. 139 (ste7) and Streptomyces sp. 139 (gtf) were cultured at $28^{\circ} \mathrm{C}$ for $96 \mathrm{~h}$ respectively. Following the protocol as described before [9], Ebosin, EPS-7 $\mathrm{m}$ and EPS-7 g were isolated from the supernatants of fermentation cultures of respective strains.

\section{Monosaccharide analysis of EPSs}

Sugar composition analysis was performed as described by Sun et al [30]. The purified polysaccharide samples (10 mg) was dissolved in $3 \mathrm{ml}$ of $2 \mathrm{M}$ TFA to hydrolyze at $115^{\circ} \mathrm{C}$ for $3 \mathrm{~h}$, then drying under a stream of nitrogen. Subsequently, the residue was treated with $200 \mu \mathrm{L}$ of $0.5 \mathrm{M} \mathrm{Na}_{2} \mathrm{CO}_{3}$ at $30^{\circ} \mathrm{C}$ for $45 \mathrm{~min}$ and added $1.5 \mathrm{~mL}$ of $4 \% \mathrm{NaBH}_{4}$ at room temperature for $2 \mathrm{~h}$. After that, the sample was neutralized with $25 \%$ acetic acid and loaded onto a cation exchange resin column eluted with $\mathrm{H}_{2} \mathrm{O}$, then discharged the excess $\mathrm{NaBH}_{4}$ with methanol. The sample residue was dried with $\mathrm{P}_{2} \mathrm{O}_{5}$ in vacuum at $85^{\circ} \mathrm{C}$ for $2 \mathrm{~h}$ and acetylated with $2.5 \mathrm{~mL}$ of acetic anhydride in $0.5 \mathrm{~mL}$ of pyridine at $100^{\circ} \mathrm{C}$ for $30 \mathrm{~min}$. After cooling, the excess reagent was discharged under a stream of nitrogen and the sample was analyzed by Gas Chromatography (GC, HP5890, HEWLETT, Houston, TX, USA). Galacturonic acid was analyzed using a method based on uronic acid carbazole reaction [31]. 


\section{Assay for IL-1R binding activity}

The enzyme-linked immune specific assay (ELISA) method reported previously was used to analyze the binding activity for IL-1R of isolated EPSs [15].

\section{Assay of the enzymatic activity for IL-1 $\beta$-converting enzyme (ICE)}

We found recently that Ebosin is an inhibitor of IL-1 $\beta$ converting enzyme [32], a key enzyme in synthesis of IL$1 \beta$. To assess the inhibitory effects of EPS-7 $m$ [16] and EPS-7 $\mathrm{g}$ on ICE enzymatic activity, the assay was conducted according to the protocol reported by koizumi et al [33]. The enzymatic reactions were performed in $100 \mu \mathrm{L}$ reaction solution consisting of $20 \mathrm{mM}$ HEPES (pH 7.5), $0.1 \mathrm{mM} \mathrm{NaCl}, 5 \mathrm{mM}$ dithiothreitol, 10\% sucrose, $250 \mu \mathrm{M}$ substrate $\mathrm{N}$-acetyl-Tyr-Val-Ala-Asp- $p$-nitroanilide, $2.0 \mathrm{IU}$ of IL-1 $\beta$-converting enzyme (ICE), $0.64 \mathrm{ng} / \mu \mathrm{L}$ and $0.128 \mathrm{ng} / \mu \mathrm{L}$ of Ebosin, EPS $-7 \mathrm{~m}$ and EPS $-7 \mathrm{~g}$ respectively at $37^{\circ} \mathrm{C}$ for $45 \mathrm{~min}$.

Enzyme-linked immunosorbent assays of IL-1 $\beta$ in FLS cells FLS was seeded at $1 \times 10^{6} / \mathrm{mL}$ in 24 -well plates and cultivated at $37^{\circ} \mathrm{C}$ for $24 \mathrm{~h}$. Ebosin $(3.2-0.128 \mathrm{ng} / \mu \mathrm{L})$ was added individually to each well and cultivated at $37^{\circ} \mathrm{C}$ for $1 \mathrm{~h}$, then FLS were stimulated with LPS $(5 \mu \mathrm{g} / \mathrm{mL}$ per well) at $37^{\circ} \mathrm{C}$ for $72 \mathrm{~h}$. IL- $1 \beta$ in the supernatants were analyzed with ELISA kits (Applygen). The cultivated FLS cells un-stimulated with LPS were as contro1, while the cultivated FLS cells stimulated with LPS but not treated by Ebosin were as control 2.

\section{Treatment of the acute inflamed mice induced by croton} oil

The mice were randomly divided into three groups: acute inflammatory mice [34], Ebosin + acute inflammatory mice and EPS $-7 \mathrm{~g}+$ acute inflammatory mice $(\mathrm{n}=8$ in each group). Ebosin and EPS-7 g (100 mg/kg) were orally administered to each mouse in the treatment groups. After $1 \mathrm{~h}, 50 \mu \mathrm{L}$ of $2 \%$ croton oil (in absolute ethyl alcohol) was smeared on the left auricle of each mouse in three groups for $4 \mathrm{~h}$. All the mice were then sacrificed and ears were excised. The degree of ear edema was measured by weighing deference between the right and left ear.

\section{Statistical analysis}

Data were presented as the mean \pm SD from at least 3 independent experiments. The significance of differences between groups was evaluated by Student's $t$-test. $p$ values less than 0.05 were considered significant.

\section{Abbreviations}

EPSs: Exopolysaccharides; FLS: Fibroblast-like synoviocytes; LAB: Lactic acid bacteria; gtf: Glucosyltransferase gene; ORFs: Open reading frames.

\section{Competing interests}

The authors declare that they have no competing interests.

\section{Authors' contributions}

ZY carried out bioactivity experiments in vitro for EPS-7 $\mathrm{g}$, EPS-7 m, Ebosin and analyzed the primary data. Sugar analysis of EPSs was performed by JS. YB and LB constructed the heterologous gene replacement strain Streptomyces sp. 139 (gtf). RJ, LG and CY isolated EPSs. GC identified anti-inflammatory activity of EPS-7 $\mathrm{g}$ and Ebosin in vivo. YL designed and conducted the experiments. YL and $R Z$ wrote and revised the manuscript. All the authors read and approved the final manuscript.

\section{Acknowledgements}

This research was supported by a grant from the Natural Science Foundation of China (NSFC 30530830). It has been supported by the Fundamental Research Funds for the Central Universities in China (2012 N09) and a grant from the National Key Project of New Drug Study of China (2012ZX09301002-001-023-02).

\section{Author details}

${ }^{1}$ Key laboratory of Biotechnology of Antibiotics, Ministry of Health, Institute of Medicinal Biotechnology, Chinese Academy of Medical Sciences \& Peking Union Medical College, Tian Tan, 100050 Beijing, China. ${ }^{2}$ Institute of Pharmacology and Toxicology, Taiping Road, 100850 Beijing, China. ${ }^{3}$ School of Biological Sciences, University of Wollongong, Wollongong, NSW 2522 Australia.

Received: 7 May 2014 Accepted: 7 July 2014

Published: 22 July 2014

\section{References}

1. Laws A, Gu Y, Marshall V: Biosynthesis, characterization and design of bacterial exopolysaccharides from lactic acid bacteria. Biotechnol Adv 2001, 19:597-625.

2. Alan DW, Lan SM: Exopolyssacharides from lactic acid bacteria: perspectives and challenges. Trends Biotechnol 2003, 21:269-274.

3. Cerning J: Exocellular polysaccharides produced by lactic acid bacteria. FEMS Microbiol Rev 1990, 7:113-130.

4. Boels IC, Ramos A, Kleerebezem M, de Vos WM: Functional analysis of the Lactococcus lactis galU and gale genes and their impact on sugar nucleotide and exopolysaccharide biosynthesis. Appl Environ Microbiol 2001, 67:3033-3040.

5. Jolly L, Vincent SJ, Duboc P, Neeser JR: Exploiting exopolysaccharides from lactic acid bacteria. Antonie Van Leeuwenhoek 2002, 82:367-374.

6. Ruas-Madiedo P, Hugenholtz J, Zoon P: An overview of the biosynthesis and applications of heteropolysaccharides from lactic acid bacteria. Int Dairy J 2002, 12:163-171.

7. Germond JE, Delley M, D'Amico N, Vincent SJ: Heterologous expression and characterization of the exopolysaccharide from Streptococcus thermophilus Sfi39. Eur J Biochem 2001, 268:5149-5156.

8. Boels IC, van Kranenburg R, Kanning MW, Chong BF, de Vos WM, Kleerebezem M: Increased exopolysaccharide production in Lactoccus lactis due to increased levels of expression of the NIZO B40 eps gene cluster. Appl Environ Microbiol 2003, 69:5029-5031.

9. Jing C, Jianbo W, Yuan L, Rong J, Baoyi L: A new IL-1 receptor inhibitor 139A: fermentation, isolation, physico-chemical properties and structure. J Antibiot 2003, 56:87-90.

10. Zhang Y, Wang LF, Bai JY, Guan MJ, Jiang R, Guo LH, Wu JB, Zhang R, Cheng GF, Li Y: Anti-inflammatory effect of Ebosin on rat collagen-induced arthritis through suppression production of interferon- $1 \beta$, interferon- 6 and tumor necrosis factor-a. Eur J Inflamm 2013, 11:677-688.

11. Wang LY, Li ST, Li Y: Identification and characterization of a new exopolysaccharide biosynthesis gene cluster from Streptomyces. FEMS Microbiol Lett 2003, 220:21-27

12. Bai LP, Jiang $R$, Shan JJ, Guo LH, Zhang Y, Zhang R, Li Y: Purification, characterization and functional analysis of asparagines synthetase encoding by ste10 gene in Ebosin biosynthesis of Streptomyces sp.139. Enzyme Microb Technol 2008, 42:548-553.

13. Qi XQ, Sun QL, Bai LP, Shan JJ, Zhang Y, Zhang R, Li Y: Identification of alpha-D-glucose-1-phosphate cytidylyltransferase involved in Ebosin 
biosynthesis of Streptomyces sp.139. Appl Microbiol Biotechnol 2009, 83:361-368.

14. Li XH, Wang L, Bai LP, Yao C, Zhang Y, Zhang R, Li Y: Cloning and characterization of a glucosyltransferase and a rhamnosyltransferase from Streptomyces sp.139. J Appl Microbiol 2010, 108:1544-1551.

15. Zhang Y, Zhou J, Chang M, Bai L, Shan J, Yao C, Li Y: Characterization and functional evidence for Ste27 of Streptomyces sp. 139 as a novel spermine/spermidine acetyltransferase. Biochem J 2012, 443:727-734.

16. Chang M, Bai LP, Shan JJ, Jiang R, Zhang Y, Guo LH, Li Y: Biochemical characteristics and function of a fucosyltransferase encoded by ste7 in Ebosin biosynthesis of Streptomyces sp. 139. J Microbiol Biotechnol 2009, 19:1092-1097.

17. Stingele F, Neeser JR, Mollet B: Identification and characterization of the eps (exopolysaccharide) gene cluster from Streptococcus thermophilus Sfi6. J Bacteriol 1996, 178:1680-1690.

18. Boels IC, Kleerebezem M, de Vos WM: Engineering of carbon distribution between glycolysis and sugar nucleotide biosynthesis in Lactoccus lactis. Appl Environ Microbiol 2003, 69:1129-1135.

19. Chabot S, Yu H-L, De Léséleuc L, Cloutier D, Van Calsteren M-R, Lessard M, Roy D, Lacroix M, Oth D: Exopolysaccharides from Lactobacillus rhamnosus RW-9595M stimulate TNF, IL-6 and IL-12 in human and mouse cultured immunocompetent cells, and IFN- in mouse splenocytes. Lait 2001, 81:683-697.

20. Hajime N, Yutaka S, Tetsuji H: Cholesterol lowering activity of rupy fermented milk. J Food Sci 1992, 57:1327-1329.

21. Joo JH, Yun JW: Structure and molecular characterization of extracellular polysaccharides produced by Trichoderma erinaceum DG-312. J Mol Biotechnol 2005, 15:1250-1257.

22. Jin $M$, Lu Z, Huang M, Wang Y: Effects of Se - enriched polysaccharide produced by Enterobacter cloacae Z0206 on alloxan - induced diabetic mice. Int J Biol Macromol 2012, 50:348-352.

23. Masja NNG, Jeroen G, Michiel $K$ : Heterologous expression of the pneumococcol serotype 14 polysaccharide in Lactococcus lactis requires lactococcal eps $A B C$ regulatory genes. Appl Environ Microbiol 2008, 74:912-915

24. Knoshaug EP, Ahlgren JA, Trempy JE: Exopolysaccharide expression in Lactococcus lactis subsp.cremoris Ropy352: evidence for novel gene organization. Appl Environ Microbiol 2007, 73:897-905.

25. Ralf RS, Claus B, Dirk R, Norbert L, Carsten JK, Joerg RW, Dagmar P: Lipopolysaccharide activates capase-1(interleukin-1-converting enzyme) in culture monocytic and endothelial cells. Blood 1998, 2:577-584.

26. Cuzzocrea S, Mazzon E, Bevilaqua C, Costantino G, Britti D, Mazzulo G: Cloricromene, a coumarine derivative, protects against collagen-induced arthritis in Lewis rats. Br J Pharmacol 2000, 131:1399-1407.

27. Sambrook J, Russell DW: Molecular cloning: a laboratory manual. 3rd edition. Cold Spring Harbor, NY: Cold Spring Harbor Press; 2001.

28. Kieser T, Bibb MJ, Chater KF, Hopwood DA: Practical Streptomyces genetics. Norwich, UK: TheJohn Innes Foundation; 2000:161-210.

29. MacNeil DJ, Gewain KM, Ruby CL, Dezeny G, Gibbons PH, MacNeil T: Analysis of Streptomyces avermitilis genes required for avermectin biosynthesis utilizing a novel integration vector. Gene 1992, 111:61-68

30. Sun QL, Wang LY, Shan JJ, Jiang R, Guo LH, Zhang Y, Zhang R, Li Y: Knockout of the gene (ste15) encoding a glycosyltransferase and its function in biosynthesis of exopolysaccharide in Streptomyces sp.139. Arch Microbiol 2007, 188:333-340.

31. Bitter T, Muir HM: A modified uronic acid carbazole reaction. Anal Biochem 1962, 4:330-334

32. Zhang Y, Guo LH, Li Y: Effect of Ebosin on the inflammatory cytokines in THP-1 cells. Acta Pharmaceutica Sinica 2013, 48:661-667.

33. Koizumi F, Matsuda Y, Nakanishi S: El-1941-1 and -2, novel interleukin-1 $\beta$ converting enzyme inhibitors produced by Farrowia sp. E-1941. J Antibiotics 2003, 56:464-469.

34. Li YT, Shen F, Bai JY, Cheng GF: Inhibition of dexamethasone, indomethacin and resveratrol on matrix methalloprotein Se-9 and the mechanism of inhibition. Acta Pharmaceutica Sinica 2003, 68:501-504.

doi:10.1186/s12934-014-0103-6

Cite this article as: Zhang et al:: Characterization of an Ebosin derivative produced by heterologous gene replacement in Streptomyces sp. 139 . Microbial Cell Factories 2014 13:103.

\section{Submit your next manuscript to BioMed Central and take full advantage of:}

- Convenient online submission

- Thorough peer review

- No space constraints or color figure charges

- Immediate publication on acceptance

- Inclusion in PubMed, CAS, Scopus and Google Scholar

- Research which is freely available for redistribution 